\title{
Comparison of analgesic efficacy of oblique subcostal and lateral transversus abdominis plane block after laparoscopic cholecystectomy
}

\author{
Keum Young So ${ }^{1,2}$, In Gook Jee ${ }^{1}$, Ki Tae Jung ${ }^{1,2}$, Sang Hun Kim ${ }^{1,2}$ \\ ${ }^{1}$ Department of Anesthesiology and Pain Medicine, Chosun University Hospital, ${ }^{2}$ Department of Anesthesiology and Pain Medicine, School \\ of Medicine, Chosun University, Gwangju, Korea
}

Received October 27, 2017

Revised November 23, 2017

Accepted December 1, 2017

Corresponding author

Sang Hun Kim

Department of Anesthesiology and

Pain Medicine, School of Medicine,

Chosun University, 309 Pilmun-

daero, Dong-gu, Gwangju 61452,

Korea

Tel: +82-62-220-3223

Fax: +82-62-223-2333

E-mail: ksh3223@chosun.ac.kr
This prospective, double-blinded, randomized, controlled study was undertaken to compare the additional postoperative analgesic effects of oblique subcostal and lateral transversus abdominis plane blocks using visual analog scale (VAS) scores and algometry determined pressure pain thresholds (PPTs), and to investigate the correlation between VAS scores and PPTs in patients on patient-controlled analgesia (PCA) after laparoscopic cholecystectomy. Sixty patients aged 20 to 65 years, ASA physical status I or II, were randomly allocated to one of three groups: a group not administered a TAP block (TAPC group, $n=20$ ), a group that received an oblique subcostal TAP block (TAPS group, $n=20$ ), and a group that received a lateral TAP block (TAPL group, $n=20$ ). Bilateral TAP blocks were performed after skin closure. All patients subsequently received fentanyl $(20 \mu \mathrm{g} / \mathrm{kg})$ PCA. At 8 hours postoperatively, VAS scores during coughing was negatively correlated with PPTs on right skin incision site $(r=-0.418, p=0.001)$. We conclude oblique subcostal and lateral TAP blocks have no additional postoperative analgesic effect in patients on PCA after laparoscopic cholecystectomy, and suggest that algometer determined PPTs offer a potential objective alternative to VAS scores for assessing postoperative pain.

Keywords: Cholecystectomy, laparoscopic; Pain, postoperative; Pressure pain threshold; Transversus abdominis plane block; Visual analog scale

\section{INTRODUCTION}

Ultrasound-guided transversus abdominis plane (TAP) blocks are being increasingly used to control postoperative pain following abdominal surgeries [1,2]. TAP blocks can be performed using several approaches, such as, the subcostal, lateral, and conventional posterior approaches [3]. These blocks are performed by injecting local anesthetic into the neurovascular plane of the abdominal wall, which is innervated by nerves from T6 to L1 $[4,5]$.
Although laparoscopic cholecystectomy is less invasive than open cholecystectomy, it is still associated with significant moderate to severe pain during the first 24 hours after surgery, especially at trocar insertion sites [6,7]. During laparoscopic cholecystectomy these sites are innervated by nerves from T6 to $\mathrm{L} 1$ at the neurovascular plane of the abdominal wall $[4,5]$, and thus, it might be expected that TAP blocks would be useful for controlling pain after laparoscopic cholecystectomy.

The visual analog scale (VAS) and numeric rating scale (NRS) are commonly used to evaluate postoperative analgesia,

This is an Open Access article distributed under the terms of the Creative Commons Attribution Non-Commercial License (http://creativecommons.org/licenses/by-nc/4.0) which permits unrestricted noncommercial use, distribution, and reproduction in any medium, provided the original work is properly cited. Copyright $($ ) Medical Biological Science and Engineering. 
though they do not objectively assess pain intensity, whereas algometer provide a more objective means of assessing pain intensity based on measurements of pressure pain threshold (PPT) and pain tolerance [8-11].

We hypothesized additional oblique subcostal or lateral TAP blocks would provide more effective analgesia than patient-controlled analgesia (PCA) alone after laparoscopic cholecystectomy, and therefore, we investigated the additional analgesic effect of oblique subcostal or lateral TAP blocks after laparoscopic cholecystectomy, and the nature of the correlation between VAS scores and PPTs.

The primary outcome measures were VAS score during coughing and PPT at 8 hours postoperatively, and the secondary outcome was the correlation between VAS scores during coughing and PPTs at 8 hours postoperatively.

\section{MATERIALS AND METHODS}

This prospective, randomized, controlled, double blinded study was approved by our Institutional Review board of Chosun University Hospital and registered with the Clinical Research Information Service (CRIS: https://cris.nih.go.kr/, ref.: KCT0001983) on July 28, 2016. Written informed consent was obtained from all participants, a legal surrogate, or the parents or legal guardians of all minors.

Sixty patients aged from 20 to 65 years of American Society of Anesthesiologists (ASA) physical status I or II, and scheduled to undergo elective laparoscopic cholecystectomy under general anesthesia were enrolled in the present study. Patients with an allergy to local anesthetics, infection at the injection site, coagulation dysfunction or bleeding tendency, a renal or hepatic function abnormality, or a mental disorder were excluded. We also excluded pregnant or breastfeeding women.

The 60 study subjects were randomly allocated to one of three groups using a random number table generated using a computer program (Fig. 1). These groups were as follows; 1) a group not provided a TAP block (TAPC group, $n=20), 2$ ) a group that received an oblique subcostal TAP block (TAPS group, $n=20$ ), and 3) a group that received a lateral TAP block (TAPL group, $\mathrm{n}=20$ ).

After premedication with intramuscular midazolam (0.05 $\mathrm{mg} / \mathrm{kg}$ ) 30 minutes before anesthesia induction, anesthesia was induced and maintained with remifentanil and propofol using a target-controlled infusion pump (Orchestra ${ }^{\circledR}$; Fresenius Vial, Brezins, France) to maintain a bispectral index score of 40 to 60 and mean arterial pressure changes within $\pm 20 \%$. Intraoperative neuromuscular block was controlled with an intermittent bolus injection of rocuronium bromide.

After skin closure, TAP blocks were performed bilaterally in each patient using a 22-gauge, $100 \mathrm{~mm}$ block needle (Disposable Nerve Blockade Needle Metal Hub, Hakko Co., Ltd., Nagano, Japan) and the M-Turbo System (SonoSite Inc., Bothwell, WA, USA). In order to eliminate inter-investigator effects, all TAP blocks were performed by one investigator.

In the TAPS group, a 13-6 MHz ultrasound transducer (SonoSite, Inc) was placed under the middle costal margin in a supine position, and rectus abdominis and transversus abdominis muscles were then identified. The needle was inserted under in-plane view. When the needle tip was placed between the posterior rectus sheath and superficial border of transversus abdominis, $0.2 \%$ ropivacaine was injected to as to cause hydrodissection within the TAP. The needle was then cautiously advanced into the hydrodissected space. Further injection and needle advancement continued along an oblique line from the xiphoid process towards the anterior part of the iliac crest, until the total volume of $0.2 \%$ ropivacaine injected reached $20 \mathrm{~mL}$ ( $40 \mathrm{mg}$ ) for each abdominal side.

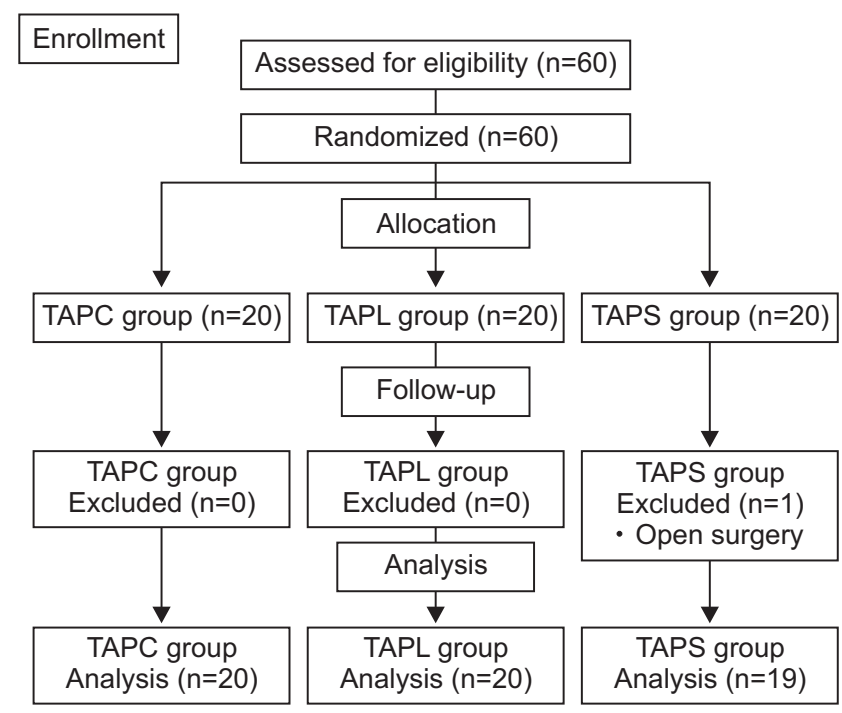

Fig. 1. CONSORT flow chart. Patients in the TAPC group did not receive a transverse abdominis plane (TAP) block, but patients in the TAPL and TAPS groups received a lateral or subcostal TAP block, respectively. TAPC group: a group not provided a TAP block, TAPL group: a group that received a lateral TAP block, TAPS group: a group that received an oblique subcostal TAP block. 
In the TAPL group, a 13-6 MHz ultrasound transducer was positioned horizontally across the abdomen. Scanning was performed from midline towards the area between the iliac crest and the costal margin, on the mid-axillary line. After identifying the external oblique, internal oblique and transversus abdominis muscles, the needle was inserted under in-plane view. When the tip of the needle was placed in the TAP between internal oblique and transversus abdominis muscles, $0.2 \%$ ropivacaine was injected so as to cause hydrodissection within the TAP. Further cautious needle advancement and repeated injections into the hydrodissected space were performed until the total injected volume of $0.2 \%$ ropivacaine reached $20 \mathrm{ml}$ ( $40 \mathrm{mg}$ ) per abdominal side.

The PCA device was primed with fentanyl $(20 \mu \mathrm{g} / \mathrm{kg}, 2$ $\mathrm{mL} / \mathrm{h}$ basal infusion rate, $2 \mathrm{~mL}$ bolus volume, and 1 minute lockout time), and was used after TAP block or after skin closure in the TAPC group.

VAS scores ( 0 to $100 \mathrm{~mm}$; 0, no pain, 100, worst imaginable pain) were measured during rest and coughing at 1, 4, 8, and 24 hours postoperatively. PPTs $\left(\mathrm{kg} / \mathrm{cm}^{2}\right)$ were measured using an algometer (Baseline ${ }^{\circledR}$ Dolorimeters; Fabrication Enterprises, White Plains, NY, USA) at 6 points (right upper, right middle, and right lower abdomen and at the three trocar insertion sites) at 1, 4, 8, and 24 hours postoperatively (Fig. 2). PPT was defined as the algometer value when patients felt least pain on increasing pressure at each measuring site. Patient ages, genders, ASA physical statuses, heights, weights, operation

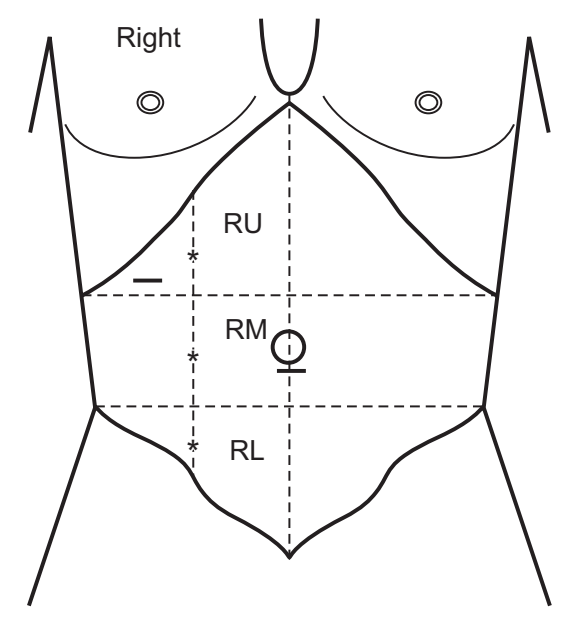

Fig. 2. Sites used to measure pressure pain threshold using an algometer. Pressure pain thresholds were measured at mid-portions of right upper, middle, and lower abdomen $\left(^{*}\right)$ and at the three trocar insertion sites (-). RU: right upper abdomen, RM: right middle abdomen, RL: right lower abdomen. and anesthesia times, intraoperative remifentanil doses infused, and fentanyl consumptions during the first 8 and 24 postoperative hours were noted.

\section{Statistical analysis}

Required sample size was determined using F-tests in G*Power software (ver. 3.1.9.1) using $\alpha$ and $\beta$ values of 0.05 and 0.2 , respectively, and an expected effect size of 0.35 , which was larger than the medium effect size (0.25) suggested by Cohen (due to lack of evidence regarding the calculation of effect size) [12]. The analysis showed 54 patients were required for the study, and thus, based on an assumed dropout rate of $10 \%$, we enrolled 60 patients.

IBM SPSS Statistics for Windows ver. 21.0 (IBM Co., Armonk, NY, USA) was used for statistical analysis. All measured values are presented as median (interquartile range), or number of patients. VAS scores and PPT values among groups were compared using the Kruskal Wallis test. In addition, oneway ANOVA followed by the Tukey's post-hoc test was used for assessing PPT values at the six abdominal points in all study subjects, which were significantly highest among them. Pearson's correlation coefficients (r) were calculated to determine the strength of relationship between VAS scores during coughing and PPTs at assessed site. Group genders and ASA physical statuses were compared using the chi-square test, and ages, heights, weights, operation and anesthesia times, intraoperative infused remifentanil doses, and postoperative fentanyl consumptions were compared using the Kruskal-Wallis test. Statistical significance was accepted for $\mathrm{p}$-values $<0.05$.

\section{RESULTS}

Fifty-nine patients were finally enrolled because the surgical plan was changed to open cholecystectomy for one patient in the TAPS group (Fig. 1).

No significant intergroup difference was observed for gender, age, height, weight, ASA physical status, operation or anesthesia time, intraoperative infused remifentanil dose, and fentanyl consumption at 8 or 24 hours postoperatively (Table 1).

VAS scores during rest and while coughing at all postoperative time points were not significantly different among the groups ( $\mathrm{p}=0.731$ and $\mathrm{p}=0.310$ at 8 hours postoperatively; Figs. 
Keum Young So, et al: Analgesia of transversus abdominis plane block

Table 1. Patient characteristics and perioperative data

\begin{tabular}{|c|c|c|c|c|}
\hline & TAPC group $(n=20)$ & TAPS group $(n=19)$ & TAPL group $(n=20)$ & $p$-value \\
\hline Gender (male/female) & $10 / 10$ & $9 / 10$ & $10 / 10$ & 0.982 \\
\hline ASA physical status (I/II) & $11 / 9$ & $11 / 8$ & $16 / 4$ & 0.197 \\
\hline Age (y) & $47.0(16.5)$ & $43.5(14.5)$ & $41.0(21.0)$ & 0.230 \\
\hline Height $(\mathrm{cm})$ & $70.5(17.5)$ & $68.0(20.0)$ & $70.0(21.0)$ & 0.744 \\
\hline Weight (kg) & $166.5(15.3)$ & $168.5(13.5)$ & $163.0(9.0)$ & 0.903 \\
\hline Operation time (min) & $30.0(22.5)$ & $35.0(18.8)$ & $45.0(30.0)$ & 0.473 \\
\hline Anesthesia time (min) & $45.0(27.5)$ & $55.0(15.0)$ & $65.0(25.0)$ & 0.053 \\
\hline Intraoperative infused remifentanil dose $(\mu \mathrm{g})$ & $425.0(200.0)$ & $500(250.0)$ & $500.0(450.0)$ & 0.549 \\
\hline Fentanyl consumption during PO 8 hours $(\mu \mathrm{g})$ & $190.0(66.8)$ & $200.0(75.0)$ & $180.0(80.0)$ & 0.736 \\
\hline Fentanyl consumption during PO 24 hours $(\mu \mathrm{g})$ & $530.0(175.0)$ & $600.0(200.0)$ & $600.0(200.0)$ & 0.697 \\
\hline
\end{tabular}

Values are presented as number only or median (interquartile range). Statistical significance was accepted for $p<0.05$. No significant differences were found between the three study groups. TAPC group: a group not provided a TAP block, TAPS group: a group that received an oblique subcostal TAP block, TAPL group: a group that received a lateral TAP block, ASA: American Society of Anesthesiologists, PO: postoperative.

A

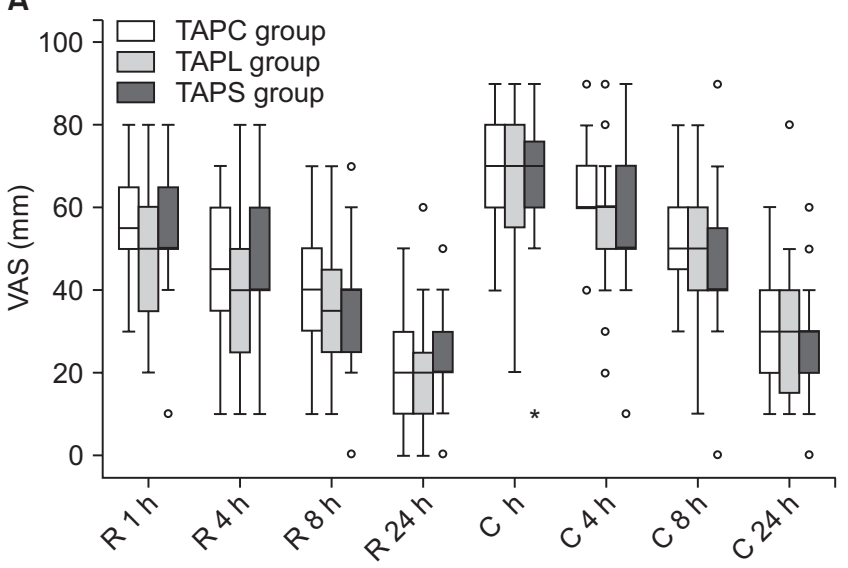

Time

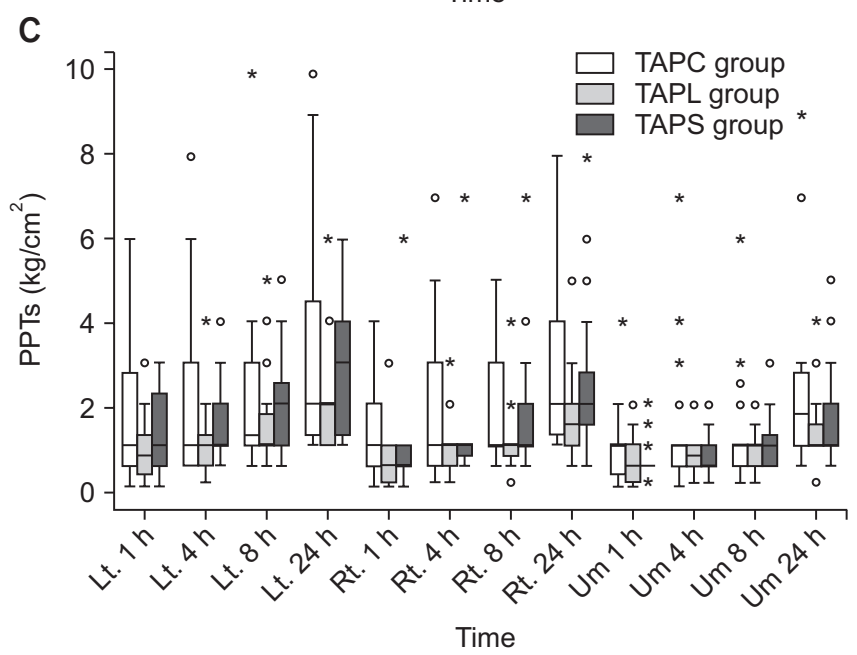

B
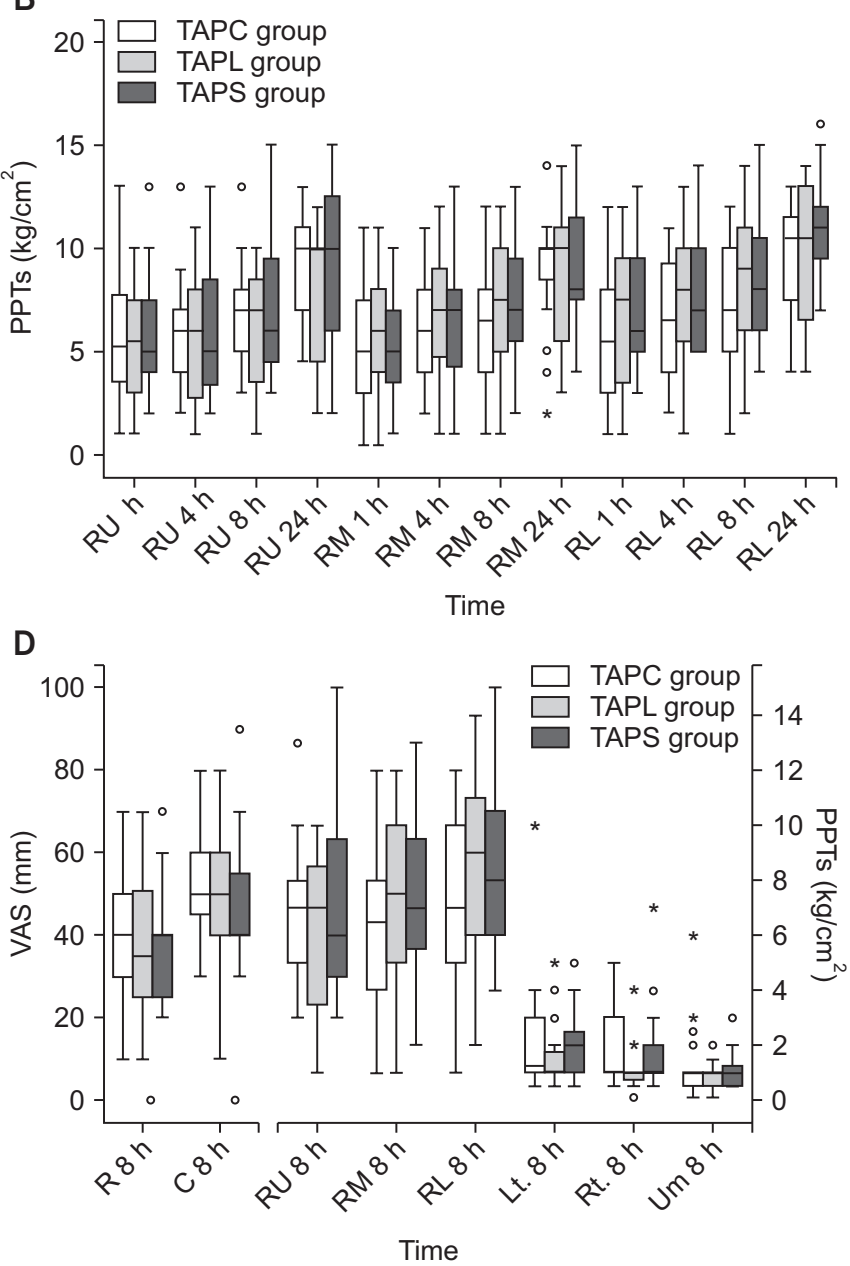

Fig. 3. Postoperative visual analog scale (VAS) scores and pressure pain thresholds (PPTs). (A) VAS scores during rest and while coughing, (B) PPTs measured on right upper, right middle, and right lower abdomens, (C) PPTs measured on the three trocar insertion points, at all postoperative time points. (D) VAS scores and PPT values at 8 hours postoperatively. TAPC group: a group not provided a TAP block, TAPS group: a group that received an oblique subcostal TAP block, TAPL group: a group that received a lateral TAP block, R: resting, C: coughing, RU: right upper abdomen, RM: right middle abdomen, RL: right lower abdomen, Lt.: left skin incision site, Rt.: right skin incision site, Um: umbilical skin incision site. 
3A and D).

PPT values on right upper, right middle, and right lower abdomens were non-significantly different among the groups at all postoperative time points $(\mathrm{p}=0.848, \mathrm{p}=0.585$, and $\mathrm{p}=$ 0.345 on right upper, right middle, and right lower abdomens at 8 hours postoperatively; Figs. 3B and D).

PPT values at the three trocar insertion sites at all postoperative time points were also non-significantly different among the groups $(\mathrm{p}=0.479, \mathrm{p}=0.100$, and $\mathrm{p}=0.685$ on left, right, and umbilical incision sites at 8 hours postoperatively; Figs. 3C and D).

In all study subjects, PPTs were non-significantly different at right upper, right middle, and right lower abdomen, and at the three trocar insertion sites at all time points (Fig. 4). PPTs at the three skin incision sites for trocar insertion were significantly lower than at non-incisional abdominal sites at all time points ( $<<0.001$, Fig. 4). Negative correlations were observed between VAS scores during coughing and PPT values at all six sites (Fig. 5). At postoperative 8 hours, the VAS during coughing showed the highest negative correlation (moderately strong) with PPT on right skin incision site ( $\mathrm{r}=$

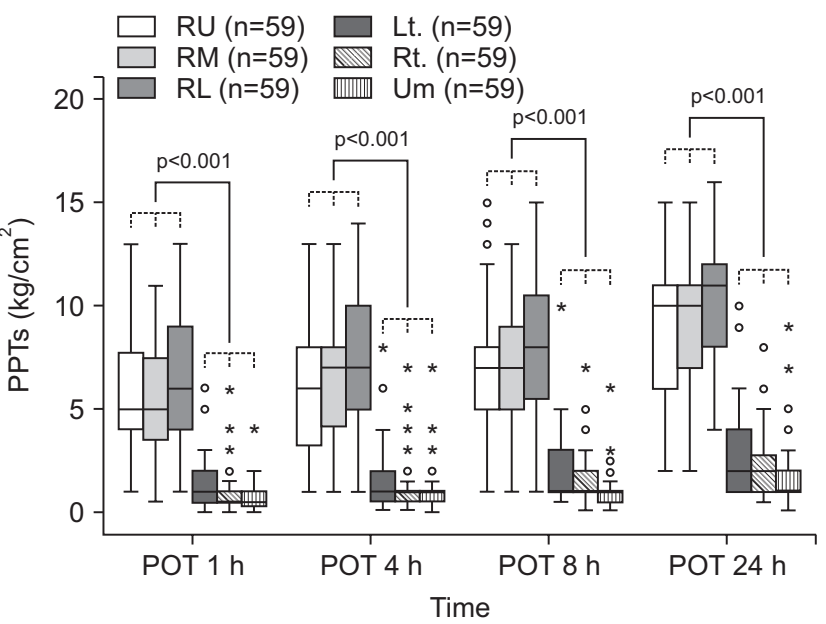

Fig. 4. Pressure pain thresholds (PPTs) at the six assessed abdominal sites at 1, 4, 8, and 24 hours after surgery in all 59 study subjects. POT: postoperative time, RU: right upper abdomen, $\mathrm{RM}$ : right middle abdomen, RL: right lower abdomen, Lt: left skin incision site, Rt: right skin incision site, Um: umbilical skin incision site. Statistical significance was accepted for $p<0.05$ for comparisons of PPTs at trocar insertion sites and PPTs at non-insertion abdominal sites (right upper, right middle, and right lower abdomen).
A

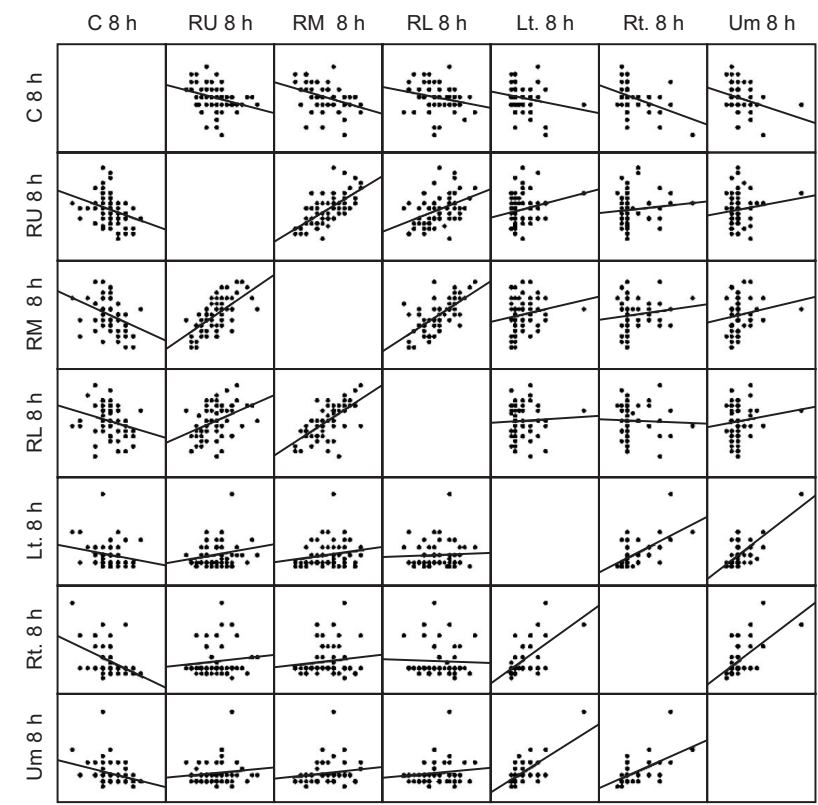

B

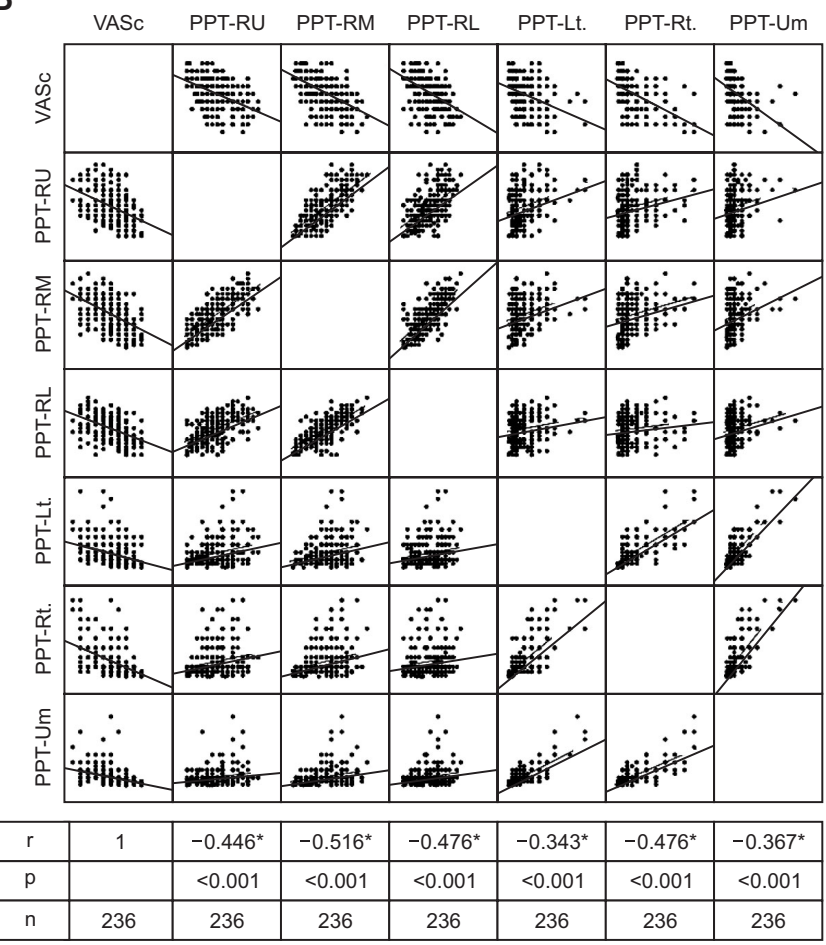

Fig. 5. Correlations between visual analog scale (VAS) scores while coughing and pressure pain thresholds (PPTs) obtained at measurement sites. (A) Correlations at 8 hours postoperatively between VAS scores and PPTs for all study subjects. (B) Correlations between VAS scores and PPTs obtained at 1, 4, 8, and 24 hours postoperatively for all study subjects and at all time points. r: Pearson correlation coefficient, p: p-value, n: number of patients, C: coughing, RU: right upper abdomen, RM: right middle abdomen, RL: right lower abdomen, Lt.: left skin incision site, Rt.: right skin incision site, Um: umbilical skin incision site, VASc: VAS during coughing. *Statistical significance was accepted for $p<0.05$. 
$-0.418, p=0.001$; Fig. $5 \mathrm{~A}$ ), and negative correlation was highest (moderately strong) on the right middle abdomen with all measured values for all study subjects and at all time points $(\mathrm{r}=-0.516, \mathrm{p}<0.001$; Fig. 5B).

\section{DISCUSSION}

This study shows neither lateral nor oblique subcostal TAP block conferred a significant additional analgesic effect above that achieved by intravenous PCA with fentanyl $(20 \mu \mathrm{g} / \mathrm{kg})$ at 8 hours postoperatively in patients underwent laparoscopic cholecystectomy. PPTs were significantly lower at the three trocar insertion sites than at the three non-incisional abdominal sites at 1, 4, 8 and 24 hours postoperatively. VAS scores during coughing were found to be moderately correlated with PPT values at right skin incision sites.

TAP blocks are peripheral nerve blocks designed to anesthetize the anterior rami of spinal nerves T6-L1, which are located between the internal oblique and transverse abdominis muscles and supply the anterior abdominal wall [13]. TAP blocks have been reported to reduce postoperative VAS, morphine consumption, and systemic analgesic requirements, and to reduce opioid-related side effects after abdominal surgeries, including laparoscopic surgery, as compared with placebo blocks [2,14-18]. Previous reports have shown TAP blocks provide significant pain relief from within 2 hours of laparoscopic surgery, but not at 24 hours postoperatively $[15,18]$. However, a meta-analysis showed TAP block provided an effective means of reducing postoperative pain during rest and of reducing opioid consumption after surgical laparoscopic procedures, but that TAP block was not superior to placebo treatment in terms of reducing early or late pain during movement [17]. As a result, the timing of significant pain relief after a TAP block remains to be determined.

The analgesic effects of TAP blocks on postoperative pain and opioid consumption probably depend on the surgical approach and trocar insertion sites used during laparoscopic surgery. We chose an oblique subcostal TAP block rather than a single subcostal TAP block, because it is considered more suitable for upper abdominal surgery, such as, laparoscopic cholecystectomy $[16,19,20]$. While single subcostal TAP block provides sensory block of dermatomes T8-T11, oblique subcostal TAP block using a multiple injection technique may provide analgesia over T6-L1 [20-22].
Bhatia et al. [23] reported subcostal TAP block was better than posterior TAP block for providing postoperative analgesia on incisions mainly involving the supra-umbilical region during laparoscopic cholecystectomy. In addition, they found the subcostal TAP group had significantly lower pain scores at 4 hours postoperatively and opioid consumptions at 24 hours postoperatively. This difference in analgesic effects may explained by nerve innervation on port site incisions for laparoscopic cholecystectomy, and differences between the dermatomal and sensory block spreads of subcostal and posterior TAP blocks [20]. The most cephalad dermatomal and sensory block spreads of subcostal and posterior TAP blocks were to T8 and T10 respectively [20]. Therefore, subcostal TAP block seems to be more effective than posterior TAP block for laparoscopic cholecystectomy because port site incisions are performed above the umbilicus, and thus, are innervated by nerves from T6 to L1 at the neurovascular plane of the abdominal wall $[4,5]$.

For the control group, we choose lateral TAP block, which has been recently used instead of posterior TAP block, although lateral TAP block mainly provide block to fewer dermatomes than posterior TAP block, which covers dermatomes T9-L1 [3,20,22,24]. A few papers compared lateral TAP block with posterior TAP block [25,26], and thus, differences between the efficacies of these blocks remain unclear, although it has been suggested posterior TAP block provides more effective postoperative pain control in patients after laparoscopic gynecologic surgery [26]. However, oblique subcostal TAP block has been reported to decrease VAS scores significantly more than lateral TAP block after laparoscopic cholecystectomy [27]. Shin et al. [28] also showed that the oblique subcostal TAP block decreased pain during rest and movement at 4 hours after surgery significantly more than lateral TAP block, and significantly more at all times after surgery than no TAP block.

Therefore, based on previous reports, we expected oblique subcostal TAP block to be more effective than lateral TAP block after laparoscopic cholecystectomy. However, this study shows that oblique subcostal TAP block did not exhibit a significant greater analgesic effect during rest or coughing than lateral TAP block in patients receiving PCA at $1,4,8$, or 24 hours postoperatively.

TAP blocks have been reported to be competitive and have additional postoperative analgesic effects when used with 
intravenous PCA $[27,29,30]$. In a previous study, TAP blocks were found to have additional analgesic effects when used with intravenous tramadol-based PCA alone during the first 24 hours after surgery, and subcostal TAP block was associated with lower VAS scores than lateral TAP block in patients that underwent laparoscopic cholecystectomy [27]. However, Guardabassi et al. [31] reported that a combination of TAP block and opioid-based PCA did not improve postoperative pain after laparoscopic hysterectomy. The present study also revealed that neither TAP block decreased postoperative pain when administered with intravenous fentanyl $(20 \mu \mathrm{g} / \mathrm{kg})$ PCA. These findings indicate that neither lateral nor oblique subcostal TAP block provide significant additional analgesic effect over intravenous PCA.

Algometer provides an objective means of evaluating PPT and pain tolerance and have been reported to provide reliable and valid assessments of pain intensity compared with subjective tools such as VAS and NRS [32,33], and VAS scores have been shown to be significantly and negatively correlated with PPTs by algometry in patients with non-surgical pain [11]. Preoperative PPTs and tolerances after fentanyl administration are useful for assessing the severity of postoperative pain and are well correlated with postoperative opioid consumption during the first 24 hours after surgery [34,35]. In addition, postoperative VAS scores are positively correlated with total PCA consumption, and negatively correlated with total intraoperative consumption of remifentanil [27]. In addition, PPTs measured by algometry are negatively related with postoperative numerical rating scale (NRS) scores, which provide a subjective means of assessing postoperative pain [36]. The present study also shows postoperative PPTs are negatively correlated with postoperative VAS scores.

In conclusion, no additional postoperative analgesic effect could be attributed to the use of subcostal or lateral TAP blocks in patients on PCA after laparoscopic cholecystectomy. Nonetheless, our findings indicate algometry determined PPTs provide a possible alternative to VAS and an objective means of assessing postoperative pain.

\section{ACKNOWLEDGEMENTS}

This study was supported by grants from the Clinical Medicine Research Institute at Chosun University Hospital (2016).

\section{CONFLICT OF INTEREST}

No potential conflict of interest relevant to this article was reported.

\section{REFERENCES}

1. Buvanendran A, Kroin JS. Multimodal analgesia for controlling acute postoperative pain. Curr Opin Anaesthesiol 2009;22:58893.

2. Peng K, Ji FH, Liu HY, Wu SR. Ultrasound-guided transversus abdominis plane block for analgesia in laparoscopic cholecystectomy: a systematic review and meta-analysis. Med Princ Pract 2016;25:237-46.

3. Hebbard P. TAP block nomenclature. Anaesthesia 2015;70:1123.

4. Rozen WM, Tran TM, Ashton MW, Barrington MJ, Ivanusic JJ, Taylor GI. Refining the course of the thoracolumbar nerves: a new understanding of the innervation of the anterior abdominal wall. Clin Anat 2008;21:325-33.

5. Petersen PL, Stjernholm P, Kristiansen VB, Torup H, Hansen EG, Mitchell AU, et al. The beneficial effect of transversus abdominis plane block after laparoscopic cholecystectomy in day-case surgery: a randomized clinical trial. Anesth Analg 2012;115:527-33.

6. Niraj G, Kelkar A, Jeyapalan I, Graff-Baker P, Williams O, Darbar A, et al. Comparison of analgesic efficacy of subcostal transversus abdominis plane blocks with epidural analgesia following upper abdominal surgery. Anaesthesia 2011;66:46571.

7. Bisgaard T, Schulze S, Christian Hjorts $\varnothing$ N, Rosenberg J, Bjerregaard Kristiansen V. Randomized clinical trial comparing oral prednisone $(50 \mathrm{mg})$ with placebo before laparoscopic cholecystectomy. Surg Endosc 2008;22:566-72.

8. Alfvén G. The pressure pain threshold (PPT) of certain muscles in children suffering from recurrent abdominal pain of nonorganic origin. An algometric study. Acta Paediatr 1993;82:4813.

9. Dahl JB, Rosenberg J, Molke Jensen F, Kehlet H. Pressure pain thresholds in volunteers and herniorrhaphy patients. Acta Anaesthesiol Scand 1990;34:673-6.

10. Tverskoy M, Oz Y, Isakson A, Finger J, Bradley EL Jr, Kissin I. Preemptive effect of fentanyl and ketamine on postoperative pain and wound hyperalgesia. Anesth Analg 1994;78:205-9.

11. Imamura M, Alfieri FM, Filippo TR, Battistella LR. Pressure pain thresholds in patients with chronic nonspecific low back pain. J Back Musculoskelet Rehabil 2016;29:327-36.

12. Cohen J. A power primer. Psychol Bull 1992;112:155-9.

13. Lissauer J, Mancuso K, Merritt C, Prabhakar A, Kaye AD, Urman RD. Evolution of the transversus abdominis plane block 
and its role in postoperative analgesia. Best Pract Res Clin Anaesthesiol 2014;28:117-26.

14. Johns N, O'Neill S, Ventham NT, Barron F, Brady RR, Daniel T. Clinical effectiveness of transversus abdominis plane (TAP) block in abdominal surgery: a systematic review and metaanalysis. Colorectal Dis 2012;14:e635-42.

15. Zhao X, Tong Y, Ren H, Ding XB, Wang X, Zong JY, et al. Transversus abdominis plane block for postoperative analgesia after laparoscopic surgery: a systematic review and metaanalysis. Int J Clin Exp Med 2014;7:2966-75.

16. El-Dawlatly AA, Turkistani A, Kettner SC, Machata AM, Delvi MB, Thallaj A, et al. Ultrasound-guided transversus abdominis plane block: description of a new technique and comparison with conventional systemic analgesia during laparoscopic cholecystectomy. Br J Anaesth 2009;102:763-7.

17. De Oliveira GS Jr, Castro-Alves LJ, Nader A, Kendall MC, McCarthy RJ. Transversus abdominis plane block to ameliorate postoperative pain outcomes after laparoscopic surgery: a meta-analysis of randomized controlled trials. Anesth Analg 2014;118:454-63.

18. Basaran B, Basaran A, Kozanhan B, Kasdogan E, Eryilmaz MA, Ozmen S. Analgesia and respiratory function after laparoscopic cholecystectomy in patients receiving ultrasoundguided bilateral oblique subcostal transversus abdominis plane block: a randomized double-blind study. Med Sci Monit 2015; 21:1304-12.

19. Hebbard PD, Barrington MJ, Vasey C. Ultrasound-guided continuous oblique subcostal transversus abdominis plane blockade: description of anatomy and clinical technique. Reg Anesth Pain Med 2010;35:436-41.

20. Lee TH, Barrington MJ, Tran TM, Wong D, Hebbard PD. Comparison of extent of sensory block following posterior and subcostal approaches to ultrasound-guided transversus abdominis plane block. Anaesth Intensive Care 2010;38:452-60.

21. Barrington MJ, Ivanusic JJ, Rozen WM, Hebbard P. Spread of injectate after ultrasound-guided subcostal transversus abdominis plane block: a cadaveric study. Anaesthesia 2009;64:74550.

22. Kehlet H. Procedure-specific postoperative pain management. Anesthesiol Clin North America 2005;23:203-10.

23. Bhatia N, Arora S, Jyotsna W, Kaur G. Comparison of posterior and subcostal approaches to ultrasound-guided transverse abdominis plane block for postoperative analgesia in laparoscopic cholecystectomy. J Clin Anesth 2014;26:294-9.

24. Bhaskar SB, Balasubramanya $\mathrm{H}$. The transversus abdominis plane block: case for optimal tap. Indian J Anaesth 2016;60:2313.

25. Abdallah FW, Laffey JG, Halpern SH, Brull R. Duration of analgesic effectiveness after the posterior and lateral transversus abdominis plane block techniques for transverse lower abdominal incisions: a meta-analysis. Br J Anaesth 2013;111: $721-35$.

26. Yoshiyama S, Ueshima H, Sakai R, Otake H. A posterior TAP block provides more effective analgesia than a lateral TAP block in patients undergoing laparoscopic gynecologic surgery: a retrospective study. Anesthesiol Res Pract 2016;2016:4598583.

27. Oksar M, Koyuncu O, Turhanoglu S, Temiz M, Oran MC. Transversus abdominis plane block as a component of multimodal analgesia for laparoscopic cholecystectomy. J Clin Anesth 2016;34:72-8.

28. Shin HJ, Oh AY, Baik JS, Kim JH, Han SH, Hwang JW. Ultrasound-guided oblique subcostal transversus abdominis plane block for analgesia after laparoscopic cholecystectomy: a randomized, controlled, observer-blinded study. Minerva Anestesiol 2014;80:185-93.

29. Chen CK, Tan PC, Phui VE, Teo SC. A comparison of analgesic efficacy between oblique subcostal transversus abdominis plane block and intravenous morphine for laparascopic cholecystectomy. A prospective randomized controlled trial. Korean J Anesthesiol 2013;64:511-6.

30. Dewinter G, Van de Velde M, Fieuws S, D'Hoore A, Rex S. Transversus abdominis plane block versus perioperative intravenous lidocaine versus patient-controlled intravenous morphine for postoperative pain control after laparoscopic colorectal surgery: study protocol for a prospective, randomized, double-blind controlled clinical trial. Trials 2014;15:476.

31. Guardabassi DS, Lupi S, Agejas R, Allub JM, Garcia-Fornari G. Efficacy of ultrasound-guided transversus abdominis plane block in laparoscopic hysterectomy. Clinical trial. Rev Esp Anestesiol Reanim 2017;64:257-61.

32. Dalamagka M, Mavrommatis C, Grosomanidis V, Karakoulas $\mathrm{K}$, Vasilakos D. Postoperative analgesia after low-frequency electroacupuncture as adjunctive treatment in inguinal hernia surgery with abdominal wall mesh reconstruction. Acupunct Med 2015;33:360-7.

33. Kinser AM, Sands WA, Stone MH. Reliability and validity of a pressure algometer. J Strength Cond Res 2009;23:312-4.

34. Hsu YW, Somma J, Hung YC, Tsai PS, Yang CH, Chen CC. Predicting postoperative pain by preoperative pressure pain assessment. Anesthesiology 2005;103:613-8.

35. Zhang H, Liu X, Jiang H, Liu Z, Zhang XY, Xie HZ. Parecoxib increases muscle pain threshold and relieves shoulder pain after gynecologic laparoscopy: a randomized controlled trial. J Pain Res 2016;9:653-60.

36. Ntritsou V, Mavrommatis C, Kostoglou C, Dimitriadis G, Tziris $\mathrm{N}$, Zagka P, et al. Effect of perioperative electroacupuncture as an adjunctive therapy on postoperative analgesia with tramadol and ketamine in prostatectomy: a randomised sham-controlled single-blind trial. Acupunct Med 2014;32:215-22. 